\title{
The importance of dementia support groups
}

\author{
Jill Walton registered general nurse, Natalie Ryan clinical research fellow, Sebastian Crutch \\ professorial research associate, Jonathan D Rohrer honorary consultant neurologist, Nick Fox \\ professor of clinical neurology
}

Dementia Research Centre, Institute of Neurology, London WC1N 3AR, UK

Robinson and colleagues refer to non-Alzheimer diagnoses of dementia. ${ }^{1}$ These less typical diagnoses pose complex problems for those with the disease and for their families, friends, and carers. When these forms of dementia affect younger people the problems can be even more challenging.

When asking the question: "What constitutes best practice in early intervention?" we must acknowledge that people living with a diagnosis of dementia generally do so in the community, supported by informal caregivers typically made up of spouses, relatives, friends, and neighbours.

Support groups have an important role to play in offering people the opportunity to acknowledge their diagnosis and its consequences alongside peers in a similar position.

Apart from the social and emotional benefits of participating, disease specific support groups enable the exchange of valuable professional and personal information and advice. They are also gateways to understanding and sharing, enabling people to cope better and for longer.

Support groups can extend to provide supportive networks across telephone, internet, and social media platforms, as well as providing the opportunity for one to one peer relationships that are continued outside of formal meetings.
Directing patients and their families to such sources of advice and support forms an important part of the post diagnosis consultation.

"Rare dementia support" offers information, advice, and support to people affected by frontotemporal dementia (behavioural variant and primary progressive aphasia), posterior cortical atrophy, familial Alzheimer's disease, and familial frontotemporal dementia.

Distinct from the personal and individual benefit to group members, support groups also fulfil an advocacy role on behalf of their members by raising awareness and representing the needs of people within the group by contributing to debates and discussions at a more strategic level.

Competing interests: None declared.

For more information visit: www.ucl.ac.uk/drc/support-groups.

Full response at: www.bmj.com/content/350/bmj.h3029/rr-0.

1 Robinson L, Tang E, Taylor J-P. Dementia: timely diagnosis and early intervention. BMJ 2015;350:h3029. (16 June.)

Cite this as: BMJ 2015;351:h3875

๑ BMJ Publishing Group Ltd 2015 